\section{The novel effect of cis-2- decenoic acid on biofilm producing Pseudomonas aeruginosa}

\author{
Vahid Soheili, ${ }^{1}$ \\ Neda Khedmatgozar Oghaz, ${ }^{1}$ \\ Zahra Sabeti Noughabi, ${ }^{2}$ Bibi Sedigheh \\ Fazly Bazzaz ${ }^{3}$ \\ 1Department of Drug and Food Control, \\ ${ }^{2}$ School of Pharmacy, ${ }^{3}$ Biotechnology \\ Research Center, School of Pharmacy, \\ Mashhad University of Medical Sciences, \\ Mashhad, Iran
}

\section{Abstract}

Microbial biofilms are a main cause of many chronic infections and mortalities, such as dental caries, cystic fibrosis, osteoradionecrosis, urinary tract infections and native valve endocarditis. These polymeric matrices are sessile communities with different rules from those forms via known planktonic bacteria. One of the important biofilm-producing human pathogens is Pseudomonas aeruginosa, which causes death in the majority of people who suffer from cystic fibrosis, AIDS, burns and neutropenic cancer. To find a method for controlling the growth and resistance of $P$. aeruginosa biofilm, this study investigated the dispersion induction of this microorganism with a diffusible signal factor (DSF), cis-2decenoic acid (CDA), in combination with Tobramycin as a useful antibiotic. Our findings confirmed that although CDA did not act as a dispersion inducer in this experiment, it did show an antimicrobial effect and decreased the MIC of Tobramycin. These results suggested that research on the probable new effects of DSF molecules will result in advances in the control of biofilm infections.

\section{Introduction}

Pseudomonas aeruginosa is an opportunistic and ubiquitous pathogen, responsible for many human infections such as nosocomial pneumonia, urinary tract infections (UTIs) and surgical wound or bloodstream infections. ${ }^{1-4}$ This common Gram-negative bacterium causes death in the majority of people who suffer from cystic fibrosis, AIDS, burns and neutropenic cancer. ${ }^{3}$ Indeed, $P$. aeruginosa is responsible for almost $10 \%$ of all hospitalacquired infections. ${ }^{5}$ It can generate a wide range of virulence factors, including extracel- lular proteases or small molecule toxins like elastase, alkaline protease, exotoxin A, rhamnolipids, pyocyanin, lectins, and HCN to overcome host defenses. ${ }^{4,6,7} P$. aeruginosa can live and reproduce in various environments as a result of its great metabolic flexibility. ${ }^{4}$ This pathogen is also resistant to the more common antibiotics because of i) its outer membrane barrier, ii) multidrug efflux pumps alongside various antibiotic-resistant genes and iii) biofilms' development of resistance to antibiotics and hosts' immune systems. ${ }^{3,5}$ Therefore, novel treatments are urgently necessary to counter $P$. aeruginosa.

Microbial biofilms are identified as an association of microorganisms that adheres by extracellular products on a surface or at some distance further than a surface. Biofilms are widespread in different environments including food fermentors, oil-well drilling pipes and ship hulls, and are connected with many infections such as dental plaque, upper respiratory infections, peritonitis, urogenital infections and diseases related to implanted medical devices. ${ }^{8}$ This city of microbes supplies a safe place for infection-producing organisms, because they form a barrier to host immune responses, phagocytosis and antibiotic therapies. Furthermore, the potentially pathogenic bacteria can grow on catheters, artificial joints, mechanical heart valves and other devices, releasing persistently from the developed biofilms and forming chronic infections. ${ }^{9}$

The variety of biofilm-producing microorganisms is fairly extensive and includes Grampositive and Gram-negative bacteria, yeasts and fungi. Some of these public-health-related pathogens are Staphylococcus aureus (including methycillin-resistant strains), Staph. epidermidis, Escherichia coli, P. aeruginosa, Burkholderia cepacia, Vibrio cholera, Candida parapsilosis and $C$. albicans. ${ }^{10}$ It has been found that biofilm forms with the virulent enhancement of such organisms. The Centers for Disease Control and Prevention (CDC) have reported that $65 \%$ of infections are caused by biofilms; similarly, the National Institutes of Health (NIH) has reported the figure as $80 \% .{ }^{9}$ These high percentages of human infections are due to the high resistance of microorganisms available in the biofilm to antimicrobial therapies. ${ }^{11}$ It has been shown that biofilm formation in $P$. aeruginosa develops through several stages, including i) attachment of the bacterium to a surface, which is reversible, ii) the subsequent irreversible association and expansion of microcolonies and iii) growth to the last stage of development, when dispersion takes place and bacteria release into the surrounding medium. ${ }^{12,13}$

It is recognized that the production of virulence factors, development of biofilm and exchange of DNA in $P$. aeruginosa are controlled by a quorum-sensing (QS) system. QS
Correspondence: Bibi Sedigheh Fazly Bazzaz, Biotechnology Research Center; School of Pharmacy, Mashhad P.0. Box: 91775-1365, Iran. Tel.: +98.51.38823252 - Fax: +98.51.38823251.

E-mail: fazlis@mums.ac.ir

Key words: Biofilms; cis-2-decenoic acid; DSF; Pseudomonas aeruginosa; Tobramycin.

Acknowledgements: We appreciatively thank Mrs Laura E. Goodin for edition of this manuscript.

Contributions: VS, conception and design of the study, data analysis, draft and critical revision of article, final approval; NKO, data acquisition, data analysis, draft and critical revision of article, final approval; ZSN, data acquisition, draft and critical revision of article, final approval; BSFB conception and design of the study; draft and critical revision of article, final approval.

Conflict of interest: the authors declare no potential conflict of interests.

Received for publication: 24 August 2015.

Revision received: 9 November 2015.

Accepted for publication: 24 November 2015.

This work is licensed under a Creative Commons Attribution NonCommercial 3.0 License (CC BYNC 3.0).

(C) Copyright V. Soheili et al., 2015

Licensee PAGEPress, Italy

Microbiology Research 2015; 6:6158

doi:10.4081/mr.2015.6158

is a global regulatory signal transduction mechanism based on the production of intercellular small molecules called autoinducers. ${ }^{4,7}$ This system allows bacteria to develop collective decisions with the mediation of autoinducers, and manages different biological functions like virulence, bacterial conjugation, bioluminescence and biofilm formation. ${ }^{1,2} \mathrm{Gram}$ negative bacteria usually use $\mathrm{N}$-acyl homoserine lactones (AHLs) as autoinducers. ${ }^{1,14}$ When these signaling molecules reach a specific concentration, which is dependent on the bacterium population, they attach to each other and activate related transcriptional factors in a cascade of regulatory procedures. ${ }^{2,14}$ of the three stages of biofilm formation, the last step - dispersion - is the most important because it provides a mechanism to manage the growth and resistance of biofilms, especially in household, medical and industrial locations. Research to discover the regulatory signals responsible for dispersion has resulted in the discovery of different chemical substances (such as $\mathrm{NaCl}$, monochloramine and high concentrations of urea), chelators (such as EDTA), surfactants (such as sodium dodecyl sulfate and Tween 20) and lysozymal enzymes, in addition to a number of antimicrobial factors. Research 
shows that the addition of such chemicals to the mixed $P$. aeruginosa and Klebsiella pneumoniae biofilms causes the elimination of some of the surface proteins and release of the infection-causing cells from the biofilms. Moreover, other studies have shown that factors such as a sudden rise in organic carbon, a rapid decrease in oxygen, starvation, the presence of prophages and nitric oxide may trigger the dispersion of aggregated bacteria. ${ }^{12}$

In the last decade, the determination of the QS molecule responsible for biofilm dispersion in the cell-cell communications has been researched extensively. Davies and Marques suggested that cis-2-decenoic acid (CDA), ${ }^{12}$ the unsaturated fatty acid formed by $P$. aeruginosa in biofilm societies, is the mediator of the dispersion response in biofilms of both Gramnegative (including $P$. aeruginosa) and Grampositive bacteria. However, when employing this signaling molecule, the resistance of microorganisms to antimicrobial therapy could be inverted as a result of the cells' transition from biofilm to the planktonic position. Therefore, the use of a dispersion agent before or along with antimicrobial agents will increase the effectiveness of these therapies by disrupting persistent biofilms.

In our present study, we investigated the effect of CDA as a dispersion inducer in combination with Tobramycin (a common antibiotic in $P$. aeruginosa infections) to determine whether it would increase the efficacy of this antimicrobial agent.

\section{Materials and Methods}

\section{Chemicals and materials}

$P$. aeruginosa ATCC 15442 was employed (American Type Culture Collection). All tests were performed in soybean casein digest broth (agar) and Mueller-Hinton broth (agar) from Himedia (India). Tobramycin and CDA were purchased from Sigma (USA). 2,3,5-triphenyltetrazolium chloride (TTC) was ordered from Merck (Germany). Statistical analysis was performed based on One-way ANOVA followed by Dunnett's t-test using SPSS software (version 16.0 ) at a significance level of 0.01 .

\section{Antibacterial activity of tobramycin}

A growth culture of $P$. aeruginosa $(24 \mathrm{~h}$ at $37^{\circ} \mathrm{C}$ ) on soybean casein digest agar (SCDA) was adjusted to $10^{6} \mathrm{CFU} / \mathrm{mL}$ with sterile normal saline (NS) (0.9\%). Different concentrations of Tobramycin $(0.5-16 \mu \mathrm{g} / \mathrm{mL})$ were prepared in Mueller-Hinton broth (MHB) by twofold serial dilution. $180 \mu \mathrm{L}$ of each concentration was placed in a 96 -well culture plate along with $20 \mu \mathrm{L}$ bacterial suspension $\left(10^{6} \mathrm{CFU} / \mathrm{mL}\right)$. Each test was performed in triplicate. Wells with only culture media and only test bacteria inoculated in MHB comprised the negative and positive control wells, respectively.

After $24 \mathrm{~h}$ incubation of the plate at $37^{\circ} \mathrm{C}$, the bacterial growth was evaluated in each well with the addition of $20 \mu \mathrm{L}$ of the colorimet- ric indicator 2,3,5-triphenyltetrazolium chloride (TTC) $(5 \mathrm{mg} / \mathrm{mL})$. Thereafter, the plates were incubated again $\left(1\right.$ hour at $37^{\circ} \mathrm{C}$ ). The minimum inhibitory concentration (MIC) was the lowest concentration of Tobramycin without any color changes. Minimum bactericidal

Table 1. The minimum inhibitory concentration (MIC) and minimum bactericidal concentration (MBC) of Tobramycin with and without cis-2-decenoic acid (CDA).

\begin{tabular}{lcc} 
& MIC $(\mu \mathrm{g} / \mathrm{mL})$ & MBC $(\mu \mathrm{g} / \mathrm{mL})$ \\
Tobramycin & 2 & 2 \\
Tobramycin and CDA $(70 \mu \mathrm{M})$ & 1 & 2 \\
\hline
\end{tabular}

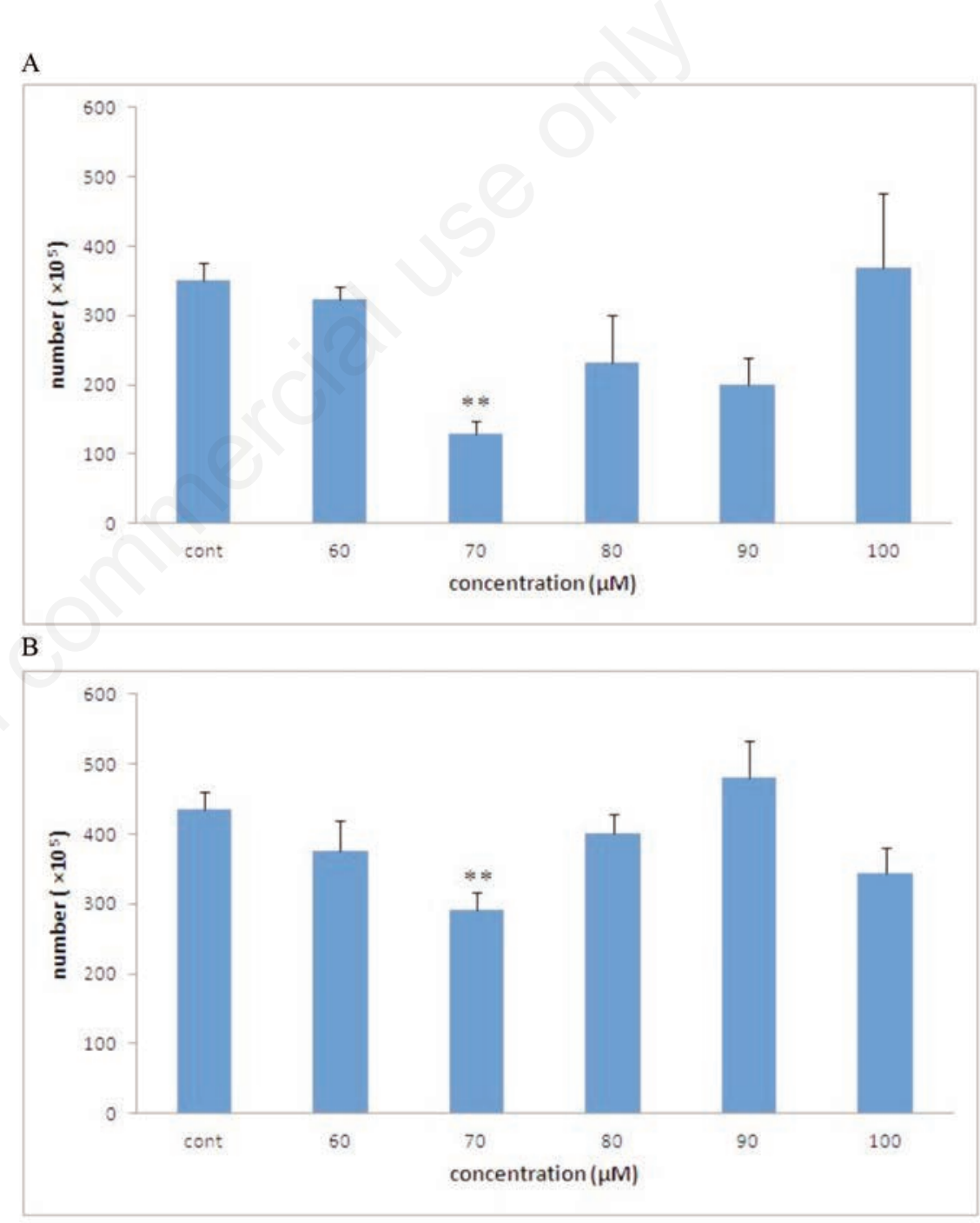

Figure 1. The effect of increasing concentrations of cis-2-decenoic acid (CDA) on planktonic cells of biofilm producer Pseudomonas aeruginosa: A) CDA was added initially B) $\mathrm{CDA}$ was added just at the last $8 \mathrm{~h}$. The results are the average of three replicates. Error bars of each concentration were represented by one standard deviation. Dunnett test was performed for all CDA samples $(60,70,80,90$ and $100 \mu \mathrm{M})$ and compared to control (first column) at $\mathrm{P}<0.01^{* *}$. 
concentration (MBC) was also studied by cultivating $20 \mu \mathrm{L}$ of no-color-change on the surface of plates containing Mueller-Hinton agar (MHA).

\section{Cis-2-decenoic acid effect on dis- persion of biofilm cells \\ Addition of cis-2-decenoic acid from the first level}

To find out the appropriate quantity of CDA in bacterial dispersion, different concentrations were prepared in MHB containing 2.5\% glucose and inoculated in a 96-well culture plate. $20 \mu \mathrm{L}$ of bacterial suspension $\left(10^{6}\right.$ $\mathrm{CFU} / \mathrm{mL}$ ) was added to each well. The test was repeated three times for each concentration. Negative and positive controls were also included.

The plate was then incubated for $96 \mathrm{~h}$ and the mixture in the wells was replaced after 24 $\mathrm{h}$ for the first day, every $12 \mathrm{~h}$ in the second and third day and every $8 \mathrm{~h}$ in the fourth day. Thereafter, the bulk-liquid of each well was removed completely, serially diluted and cultured on SCDA. Biofilms attached to the well were also removed by fully scratching the well surface in the presence of $100 \mu \mathrm{L}$ sterile NS and transmitted separately to SCDA containing plates. All plates were incubated at $37^{\circ} \mathrm{C}(24 \mathrm{~h})$. Dispersion induction was assessed by counting colonies obtained from the bulk-liquid and biofilm portion of each well.

\section{Addition of cis-2-decenoic acid in the final $8 h$}

This experiment was also repeated in the presence of different concentrations of CDA, added to the wells only in the final $8 \mathrm{~h}$ of media replacement. Other conditions were as before. Similarly, the resulting colonies from each well were counted.

\section{Antibacterial activity of cis-2- decenoic acid in combination with tobramycin}

The most effective concentration of CDA was analyzed in combination with Tobramycin. The test stages were shown to be the same as in the MIC determination. Briefly, the resulting MIC of Tobramycin was prepared again and various concentrations of the antibiotic were provided according to the two-fold serial dilution method. From each concentration, $180 \mu \mathrm{L}$ was inoculated in a 96-well culture plate to which was added a $20 \mu \mathrm{L}$ bacterial suspension $\left(10^{6} \mathrm{CFU} / \mathrm{mL}\right)$

The MIC was determined after $24 \mathrm{~h}$ incubation of the plate at $37^{\circ} \mathrm{C}$ by addition of TTC, and the $\mathrm{MBC}$ was defined by cultivation of no-colorchange cultures.

\section{Results}

The antibacterial activity of Tobramycin against the tested bacteria is presented in Table 1, which showed that in this case, the MIC is equal to the MBC.

In the next step, CDA efficacy was studied in two ways. Firstly, CDA was added from beginning of the experiment to the bacterial culture in low levels $(2.5,5$, and $10 \mathrm{nM})$. Table 2 summarizes the average numbers of colony-forming units (CFU) resulting from the upper phase of formed biofilms. The results indicated that the employed concentrations of CDA were not enough to disperse bacteria from biofilm polymeric matrices. Therefore, upper concentrations $(60,70,80,90$ and $100 \mu \mathrm{M})$ were prepared and the experiment was repeated the same as previous. The results were presented in Table 3. Once more, no effect was observed compared with the positive control. However, the average number of microbial colonies in the well containing $70 \mu \mathrm{M}$ CDA decreased significantly $(\mathrm{P}<0.01)$ in the upper phase (Figure 1A).

Secondly, CDA was added just in the final 8 $h$ of incubation period. The other stages of the test were completed as before. Again, no significant difference in the cell dispersion was achieved, but the average number of colonies in the upper phase decreased considerably $(\mathrm{P}<0.01)$ in the presence of $70 \mu \mathrm{M}$ CDA (Table 3 and Figure 1B).

Table 2. Average number of CFUs of Pseudomonas aeruginosa in presence of cis-2-decenoic Acid (CDA) at concentrations of 2.5, 5, and $10 \mathrm{nM}$.

\begin{tabular}{|c|c|c|c|c|}
\hline \multirow[b]{2}{*}{ CDA conc. (nM) } & \multicolumn{2}{|c|}{ CDA added initially } & \multicolumn{2}{|c|}{ CDA added in the last $8 \mathrm{~h}$} \\
\hline & $\begin{array}{l}\text { Average N. of planktonic } \\
\text { cells }\left(\times 10^{5}\right)\end{array}$ & $\begin{array}{l}\text { Average No. of biofilm } \\
\text { cells }\left(\times 10^{5}\right)\end{array}$ & $\begin{array}{c}\text { Average No. of planktonic } \\
\text { cells }\left(\times 10^{5}\right)\end{array}$ & $\begin{array}{l}\text { Average No. of biofilm } \\
\text { cells }\left(\times 10^{5}\right)\end{array}$ \\
\hline 0 & 380 & 230 & 360 & 235 \\
\hline 2.5 & 420 & 250 & 220 & 290 \\
\hline 5 & 320 & 277 & 170 & 270 \\
\hline 10 & 260 & 119 & 350 & 125 \\
\hline
\end{tabular}

Table 3. Average number of CFUs of Pseudomonas aeruginosa in presence of cis-2-decenoic Acid (CDA) at concentrations of 60, 70, 80,90 and $100 \mu \mathrm{M}$.

\begin{tabular}{|c|c|c|}
\hline & CDA added initially & CDA added in the last $8 \mathrm{~h}$ \\
\hline CDA conc. (nM) & $\begin{array}{cc}\begin{array}{c}\text { Average N. of planktonic } \\
\text { cells }\left(\times 10^{5}\right)\end{array} & \begin{array}{c}\text { Average No. of biofilm } \\
\text { cells }\left(\times 10^{5}\right)\end{array}\end{array}$ & $\begin{array}{l}\text { Average No. of planktonic } \\
\text { cells }\left(\times 10^{5}\right)\end{array}$ \\
\hline
\end{tabular}

\begin{tabular}{lllll}
0 & 350 & 170 & 435 & 330 \\
60 & 323 & 180 & 375 & 353 \\
\hline 70 & 130 & 147 & 292 & 315 \\
80 & 233 & 212 & 400 & 335 \\
\hline 90 & 200 & 430 & 480 & 410 \\
100 & 370 & 400 & 345 & 361
\end{tabular}


To investigate the possible antimicrobial effect of CDA, the concentrations of $70 \mu \mathrm{M}$ were tested in combination of Tobramycin. As shown in Table 1, CDA reduced the MIC of Tobramycin to half of its previous value. However, the MBC of this antibiotic remained unchanged.

\section{Discussion}

Tobramycin, an aminoglycoside antibiotic derived from Streptomyces tenebrarius, operates through disrupting protein synthesis. This mechanism results in enhancement of the cell membrane's permeability, increasing disruption of the microorganism envelope and finally the death of the bacteria. It is confirmed that Tobramycin acts against Gram-negative bacteria such as $P$. aeruginosa and has bactericidal effects at concentrations identical to or somewhat greater than those of MIC. ${ }^{15,16}$

It has been confirmed that unlike planktonic cells the microorganisms within biofilms are not susceptible to the antibiotics. ${ }^{17}$ This resistance disappeared after the alteration of biofilm cells to a planktonic growth condition. The inherent tolerance of microbial biofilms to antibiotics has resulted in the troubles with their eradication and control of patients with such infections. ${ }^{18}$ For example, antibiotic therapy in cystic fibrosis patients with $P$. aeruginosa pneumonia often provides somewhat relief from symptoms, but it cannot treat the infection. ${ }^{19}$ The reason for this variation in antibiotic susceptibility between the same cells in planktonic and biofilm forms may possibly be due to differences in the antibiotic diffusion or complex alterations in the microbial physiology of the biofilm. ${ }^{18}$

Numerous studies have defined different fatty acids as signaling molecules - named diffusible signal factors (DSF) - in bacterial communication. ${ }^{12,20-24}$ For example, eight fatty acids that aid translocation of the bacteria and communication between $P$. aeruginosa cells have been separated from Stenotrophomonas maltophila. ${ }^{23,25}$ Another fatty acid, cis-2-dodecenoic acid, facilitates the communication between $B$. cenocepacia and $C$. albicans. Therefore it seems that DSFs, like AHLs, have a special role in cell-cell interaction. ${ }^{23}$

One of these DSFs is CDA (Figure 2); a dispersion autoinducer which has been demonstrated that is effective in biofilm dispersion of not only $P$. aeruginosa, but also Streptococcus mutans, E. coli, K. pneumoniae, Proteus mirabilis, Strep. pyogenes, Bacillus subtilis, Staph. aureus, and the yeast C. albicans. ${ }^{23}$ Since Davies and Marques suggested that the minimum concentration of CDA for dispersion induction of biofilms formed by $P$. aeruginosa PA01 was $2.5 \mathrm{nM},{ }^{12}$ this concentration was selected as the lowest concentration, and the effect of the molecule was evaluated at 2.5, 5 and $10 \mathrm{nM}$.

In this experiment, CDA efficacy was investigated in two different ways: addition from the first level and only for an eight hour period. We hypothesized this might result in cell dispersion and reduce biofilm formation. As a result, the planktonic bacterial density would increase. At the same time, biofilm cells would decrease in contrast to the positive control. These two requisites were considered for selecting an appropriate concentration of CDA.

As the two declared requisites, compared with the positive control, were not satisfied in different concentrations of CDA (Table 2), the test was repeated with higher quantities. Again, no dispersing effect was detected in the presence of various concentrations of CDA but surprisingly, the microbial colonies in the well containing $70 \mu \mathrm{M}$ CDA (either from the first level or just in the final 8 hours of incubation period), decreased significantly $(\mathrm{P}<0.01)$ in the upper phase (Table 3 and Figure 1).

In this experiment, plate-counting was used instead of measurements of released cells' optical density (OD), which is used by Davies and Marques. ${ }^{12}$ Plate-counting is a good method for the study of bacterial growth. It is also useful when examining the effects of different factors on bacterial cultures. We should note that OD measurement techniques cannot determine the size of bacterial colonies. Thus plate-counting will remain an essential approach for validating new techniques to study growth factors. ${ }^{26}$ These findings are also different from the observations of Davies and Marques,${ }^{12}$ who reported that CDA was capable of dispersion induction in established biofilm micro-colonies after being added exogenously to $P$. aeruginosa $\mathrm{PA01}$ biofilms at the native concentration of $2.5 \mu \mathrm{M}$. They also believed that the cis isomer of 2-decenoic acid was active in the range of $1.0 \mathrm{nM}$ to $10 \mathrm{mM}$. Additionally, according to their findings, exposure of $P$. aeruginosa PA01 biofilms with dispersion inducer at the final hour of incubation resulted in complete micro-colony disaggregation. ${ }^{12}$ The results of colony-counting showed no difference between the tests and positive control. Furthermore, the addition of CDA from the first or in the final $8 \mathrm{~h}$ of incubation cannot induce dispersion.

The diversity in the current study's results may be the result of strain variations in $P$. aeruginosa. In fact, inter-species genome diversity could be the reason for these different responses. However, this diversity in decenoic-acid activity has been seen before in another decenoic-acid derivative, 10-Hydroxy2-decenoic acid. In an experiment, this unsaturated fatty acid, which is extracted from royal jelly, caused an effective reduction in the adherence of Streptococcus mutans, ${ }^{27}$ the com- mon flora of mouth and the major etiological cause of dental caries, ${ }^{28}$ while in another study this molecule showed antimicrobial effects on some microorganisms, including Lactobacillus brevis and Pediococcus damnosus. ${ }^{29}$ In our experiment, concentrations higher than 100 $\mu \mathrm{M}$ were not tested.

As shown in Table 1, CDA decreased the MIC of Tobramycin to $50 \%$ at the critical concentration of $70 \mu \mathrm{M}$. The other prepared concentrations of this signaling factor were not tested in combination with Tobramycin. The reason for this antibacterial outcome in the presence of only $70 \mu \mathrm{M}$ CDA could not be determined, but some kinds of antiseptics, such as alcohol, act in the same way. More investigations are necessary to find out whether CDA has dispersion or antimicrobial effects on different strains of P. aeruginosa.

\section{Conclusions}

The results of the current work enhance knowledge about DSFs' role in $P$. aeruginosa and provided different effects for CDA. In other words, our findings indicated that CDA, which performs as a dispersion inducer in $P$. aeruginosa PA01, has antibacterial properties against another strain, $P$. aeruginosa ATCC 15442. These outcomes can facilitate the development of new methods to increase the effectiveness of Tobramycin against $P$. aeruginosa, especially in cystic-fibrosis patients.

\section{References}

1. Ding X, Yin B, Qian L, et al. Screening for novel quorum-sensing inhibitors to interfere with the formation of Pseudomonas aeruginosa biofilm. J Med Microbiol 2011;60:1827-34.

2. Dekimpe V, Déziel E. Revisiting the quorum-sensing hierarchy in Pseudomonas aeruginosa: the transcriptional regulator RhIR regulates LasR-specific factors. Microbiology 2009;155:712-23.

3. Bottomley MJ, Muraglia E, Bazzo R, et al. Molecular insights into quorum sensing in the human pathogen Pseudomonas aeruginosa from the structure of the virulence regulator LasR bound to its autoinducer. $\mathrm{J}$ Biol Chem 2007;282:13592-600.

4. Croda-García G, Grosso-Becerra V, Gonzalez-Valdez A, et al. Transcriptional regulation of Pseudomonas aeruginosa rhlR: role of the CRP orthologue Vfr (virulence factor regulator) and quorum-sensing regulators LasR and RhIR. Microbiology 2011;157:2545-55.

5. Zou Y, Nair SK. Molecular basis for the 
recognition of structurally distinct autoinducer mimics by the Pseudomonas aeruginosa LasR quorum-sensing signaling receptor. Chem Biol 2009;16:961-70.

6. Dubern J-F, Diggle SP. Quorum sensing by 2-alkyl-4-quinolones in Pseudomonas aeruginosa and other bacterial species. Mol Biosyst 2008;4:882-8.

7. Yu S, Jensen V, Seeliger J, et al. Structure elucidation and preliminary assessment of hydrolase activity of PqsE, the Pseudomonas quinolone signal (PQS) response protein. Biochemistry-US 2009;48:10298-307.

8. Reid G. Biofilms in infectious disease and on medical devices. Int $\mathrm{J}$ Antimicrob Agents 1999;11:223-6.

9. Sawhney R, Berry V. Bacterial biofilm formation, pathogenicity, diagnostics and control: an overview. Indian J Med Sci 2009;63:313-21.

10. Donlan RM. Role of biofilms in antimicrobial resistance. ASAIO J 2000;46:S47-52.

11. Prasanna S, Doble M. Medical biofilms: its formation and prevention using organic molecules. J Indian Inst Sci 2012;88:27-35.

12. Davies DG, Marques CN. A fatty acid messenger is responsible for inducing dispersion in microbial biofilms. J Bacteriol 2009;191:1393-403.

13. Sihorkar V, Vyas S. Biofilm consortia on biomedical and biological surfaces: delivery and targeting strategies. Pharm Res 2001;18:1247-54.

14. Zeng Z, Qian L, Cao L, et al. Virtual screening for novel quorum sensing inhibitors to eradicate biofilm formation of
Pseudomonas aeruginosa. Appl Microbiol Biot 2008;79:119-26.

15. McKeage K. Tobramycin inhalation powder: a review of Its use in the treatment of chronic Pseudomonas aeruginosa infection in patients with cystic fibrosis. Drugs 2013;73:1815-27.

16. Novartis Pharmaceuticals Corporation. Tobi Podhaler (Tobramycin inhalation powder). Full prescribing information. Available from: www,pharma.us.novartiscom.product/pi/pdf/tobipodhaler.pdf. 2013; Accessed 17 Oct 2013.

17. Stewart PS. Theoretical aspects of antibiotic diffusion into microbial biofilms. Antimicrob Agents Chemother 1996;40:2517-22.

18. Ceri H, Olson M, Stremick C, et al. The calgary biofilm device: new technology for rapid determination of antibiotic susceptibilities of bacterial biofilms. J Clin Microbiol 1999;37:1771-6.

19. Costerton J, Stewart PS, Greenberg E. Bacterial biofilms: a common cause of persistent infections. Science 1999;284:131822.

20. Ryan RP, Dow JM. Communication with a growing family: diffusible signal factor (DSF) signaling in bacteria. Trends Microbiol 2011;19:145-52.

21. Huang CB, Alimova Y, Myers TM, et al. Short-and medium-chain fatty acids exhibit antimicrobial activity for oral microorganisms. Arch Oral Biol 2011;56:650-4.

22. Twomey KB, O'Connell OJ, McCarthy Y, et al. Bacterial cis-2-unsaturated fatty acids found in the cystic fibrosis airway modulate virulence and persistence of Pseudomonas aeruginosa. ISME J 2011;6:939-50.

23. Estrela AB, Abraham WR. Combining biofilm-controlling compounds and antibiotics as a promising new way to control biofilm infections. Pharmaceuticals 2010; 3:1374-93.

24. Ryan RP, Dow JM. Diffusible signals and interspecies communication in bacteria. Microbiology 2008;154:1845-58.

25. Huang TP, Lee Wong AC. Extracellular fatty acids facilitate flagella-independent translocation by Stenotrophomonas maltophilia. Res Microbiol 2007;158:702-11.

26. Biesta-Peters EG, Reij MW, Joosten H, et al. Comparison of two optical-densitybased methods and a plate count method for estimation of growth parameters of Bacillus cereus. Appl Environ Microb 2010;76:1399-405.

27. Yousefi B, Ghaderi S, Rezapoor-Lactooyi A, et al. Hydroxy decenoic acid down regulates gtfB and gtfC expression and prevents Streptococcus mutans adherence to the cell surfaces. Ann Clin Microbiol Antimicrob 2012;11:1-7.

28. Tahmourespour A, Nabinejad A, Shirian H, et al. The comparison of proteins elaborated by Streptococcus mutans strains isolated from caries free and susceptible subjects. Iran J Basic Med Sci 2013;16:656-60.

29. Li S, Jiang Z, Thamm L, et al. 10-Hydroxy2-decenoic acid as an antimicrobial agent in draft keg-conditioned wheat beer. J Am Soc Brew Chem 2010;68:114-8. 